\title{
LA LEISHMANIOSE CANINE EN ITALIE DE 1910 A 1983
}

\author{
E. POZIO, L. GRADONI et M. GRAMICCIA
}

RÉSUMÉ. La leishmaniose canine a été signalée pour la première fois en Italie par Basile (1910). Depuis lors, de nombreux cas ont été rapportés.

Pour avoir un tableau général de la répartition de la leishmaniose eanine de 1910 à nos jours, nous avons recueilli tous les cas de Chiens leishmaniens qui ont été enregistrés dans la littérature, complétés par des données personnelles.

Une carte qui tient compte de l'origine des cas montre :

a) l'actuelle distribution de la leishmaniose canine en Italie centrale, dans le sud et dans les îles ;

b) la persistance de la leishmaniose canine dans quelques foyers depuis 70 années ou plus ;

c) l'augmentation du nombre des nouveaux foyers d'infection signalés depuis les années précédant la guerre jusqu'à nos jours.

Contrairement à la plus large diffusion de la leishmaniose canine, il y a eu en Italie une réduction marquée des cas humains de la leishmaniose cutanée et viscérale, phénomène qui a été observé également dans les autres pays de la Méditerranée. Les auteurs discutent les données récentes concernant l'épizootie de la leishmaniose canine.

\section{Canine leishmaniasis in Italy from 1910 to 1983.}

SUMMARY. Canine leishmaniasis has first been recorded in Italy by Basile (1910). Since then, many cases have been reported.

In order to have a general picture of canine leishmaniasis distribution in Italy, from 1910 up to present times, we have collected all records of leishmaniotic dogs which have appeared in the literature, integrated by personal data.

A map has been drawn, according to the origin of cases, showing :

a) the present distribution of canine leishmaniasis in central and southern Italy and its islands ;

b) the persistence of canine leishmaniasis in some foci for the last 70 years or more ;

c) the increasing trend of new foci reports from the pre-war to present times.

As opposed to the high diffusion of canine leishmaniasis there has been, in Italy, a marked reduction of human cases of visceral and cutaneous leishmaniasis, a phenomenon which has been observed in other Mediterranean countries as well. Recent data on the epizonlogy of canine leishmaniasis are discussed.

Laboratorio di Parassitologia, Istituto Superiore di Sanità, Viale Regina Elena, 299, 00161 Rome, Italie Accepté le 9 novembre 1984 . 


\section{Introduction}

La leishmaniose canine décrite pour la première fois en Italie par Basile (1910), a été l'objet d'une abondante bibliographie, ce qui indique une vaste diffusion de la maladie dans ce pays.

Certains auteurs italiens avaient déjà mis en relief le rôle probable du Chien comme réservoir de la leishmaniose viscérale humaine (Basile, 1910 ; Sangiorgi, 1911), bien que ce ne fût que plus tard que les premières preuves confirmant cette thèse furent obtenues, lorsque Adler et Theodor (1935a et b) démontrèrent que le parasite pouvait être transmis par les phlébotomes du Chien à l'Homme, et lorsque Gramiccia et coll. (1982) montrèrent l'identité des parasites isolés du Chien et de l'Homme.

Dans ce travail, nous avons recueilli tous les renseignements bibliographiques relatifs à la leishmaniose canine, pour rédiger une carte sur la répartition de cette maladie en Italie. La majeure partie de ces renseignements a été obtenue de la bibliographie italienne sur les leishmanioses de Pampiglione et Bettini (1981) et a été complétée avec des travaux très récents et nos données personnelles.

\section{Résultats et discussion}

Le tableau I montre tous les renseignements tirés de la littérature sur la présence de la leishmaniose canine en Italie, depuis 1910 jusqu'en 1983, et de nos données personnelles recueillies dans différents foyers de 1976 à maintenant. Chaque information a été analysée pour connaître la région et la localité d'origine. On a aussi indiqué : le type de test utilisé pour le diagnostic, le nombre de Chiens positifs sur le nombre de Chiens examinés en cas d'enquêtes épidémiologiques et les cas isolés éventuels de leishmanioses qui ont été obtenus. Un seul renseignement bibliographique, concernant un Chien errant infecté sur 310 Chiens examinés il y a plus de 70 ans à Turin (Sangiorgi, 1911), n'a pas été inclus dans ce tableau. Dans les foyers de la Toscane, de l'Ombrie, quelques-uns des Pouilles (provinces de Brindisi et Lecce) et de la Basilicate, nous n'avons que des renseignements récents. Au contraire, pour Rome, Messine, Catane, Palerme et Sassari, nous avons une étude presque continue des cas pendant 70 ans, ce qui indique que beaucoup de foyers n'ont pas disparu avec l'urbanisation, mais ont été seulement déplacés par la progression des limites des villes.

Les informations tirées de la littérature concernent :

- des cas particuliers signalés par les vétérinaires (l'anamnèse manque souvent, ce qui fausse notablement la véritable diffusion de la maladie);

- des enquêtes effectuées sur des Chiens errants, auprès des fourrières (quelques travaux, comme celui de Basile, 1910, à Bordonaro (Messine)). La fréquence d'infection est certainement élevée si l'on tient compte du fait que les autopsies n'ont été effectuées que sur les Chiens présentant des signes évidents de la maladie; 
Tableau I. - Cas de leishmaniose canine signalés en Italie de 1910 à 1983.

\begin{tabular}{|c|c|c|c|}
\hline $\begin{array}{l}\text { Région } \\
\quad \text { Localité }\end{array}$ & $\begin{array}{l}\text { Nombre de } \\
\text { chiens } \\
\text { positifs* }\end{array}$ & $\begin{array}{l}\text { Type } \\
\text { de } \\
\text { test** }\end{array}$ & Bibliographie \\
\hline \multicolumn{4}{|l|}{ Frioul-Vénétie Julienne } \\
\hline Trieste & $9 / 115$ & $\mathrm{P}$ & Vlachi, 1936 \\
\hline \multicolumn{4}{|l|}{ Émilie-Romagne } \\
\hline Bologne & $1 / 280$ & $\mathrm{P}$ & Franchini, 1925 \\
\hline & & $\mathrm{P}$ & Di Domizio, 1955 \\
\hline & $132 / 8454$ & $\mathrm{~S} * * *$ & Pampiglione et coll., 1974 \\
\hline Modène & 2 & $\mathrm{P}$ & Serra, 1941 \\
\hline \multicolumn{4}{|l|}{ Toscane } \\
\hline Florence et la province & $137 / 611$ & $\mathrm{~S}(7 \mathrm{P}) !$ & Palarchi et coll., 1979 \\
\hline - $\quad-$ & 41 & $\mathrm{~S}$ & \\
\hline Baccinello - & $\begin{array}{c}41 \\
3 / 103\end{array}$ & $S(1 P) !$ & $\begin{array}{l}\text { 1.Z.L.T., 1981 } \\
\text { Gradoni et coll., } 1980\end{array}$ \\
\hline Orbetello Scalo & 1 & P. S! & Gramiccia et coll., 1983 \\
\hline Monte Argentario & $41 / 171$ & $S(12 P) !$ & Pozio et coll., 1981a \\
\hline & & & \\
\hline \multicolumn{4}{|l|}{ Ombrie } \\
\hline Terni & $5 / 100$ & S & Polidori et coll., 1980 \\
\hline \multicolumn{4}{|l|}{ Abruzzes } \\
\hline Chieti (province) & 1 & $\mathrm{P}$ & Venturoli, 1982 \\
\hline Pescara & 2 & $\mathrm{P}$ & Silvagni, 1955 \\
\hline Moscufo & 3 & $\mathrm{~S}(1 \mathrm{P}) !$ & Données des auteurs \\
\hline Teramo & 1 & $\mathrm{P}$ & Galassi, 1953 \\
\hline - & 3 & $\mathrm{P}$ & Silvagni, 1955 \\
\hline Roseto degli Abruzzi & 1 & $\mathrm{P}$ & Silvagni, 1955 \\
\hline \multicolumn{4}{|l|}{ Latium } \\
\hline Rome & $16 / 60$ & $\mathrm{P}$ & Basile, 1910 \\
\hline- & $4 / 380$ & $\mathrm{P}$ & Marchesi et coll,. 1934 \\
\hline - & $18 / 861$ & $\mathrm{P}$ & Marchesi et coll., 1935 \\
\hline — & $33 / 133$ & $\mathrm{~S}(2 \mathrm{P}) !$ & Lillini et coll., 1979 \\
\hline — & 49 & & I.Z.L.T., 1980 \\
\hline - & 23 & $\mathrm{~S}$ & I.Z.L.T., 1981 \\
\hline Anzio & 2 & $\mathrm{~S}$ & Données des auteurs \\
\hline Civitavecchia & 1 & $\mathrm{P}$ & Zechini, 1942 \\
\hline - & 2 & $\mathrm{~S}$ & Lillini et Cavallina, comm. pers. \\
\hline Fondi & $4 / 53$ & $\mathrm{~S}$ & Amalfitano et coll., 1948 \\
\hline \multicolumn{4}{|l|}{ Molise } \\
\hline Campobasso & 1 & $P$ & Mastropietro, 1953 \\
\hline
\end{tabular}


TABLEAU I (suite)

\begin{tabular}{|c|c|c|c|}
\hline $\begin{array}{l}\text { Région } \\
\text { Localité }\end{array}$ & $\begin{array}{l}\text { Nombre de } \\
\text { chiens } \\
\text { positifs* }\end{array}$ & $\begin{array}{c}\text { Type } \\
\text { de } \\
\text { test** }\end{array}$ & Bibliographie \\
\hline \multicolumn{4}{|l|}{ Campanie } \\
\hline Naples & $18 / 439$ & $\mathrm{P}$ & Lucrezi, 1940 \\
\hline- & 1 & $\mathrm{P}$ & Persechino et Agresti, 1973 \\
\hline - & 1 & $\mathrm{P}$ & Von Schlotke, 1975 \\
\hline Boscoreale, Boscotrecase & $4 / 59$ & $\mathrm{P}$ & Albano, 1941 \\
\hline Bagnoli & $2 / 30$ & $\mathrm{~S}$ & Données des auteurs \\
\hline Praiano & 1 & $\mathrm{P}, \mathrm{S} !$ & - - - \\
\hline S. Marco di Castellabate & 1 & P. S! & $-\quad-$ \\
\hline \multicolumn{4}{|l|}{ Pouilles } \\
\hline Foggia & $3 / 102$ & $\mathrm{P}$ & Nardi et coll., 1952 \\
\hline - & $3 / 120$ & $\mathrm{P}$ & Corradetti et coll., 1956 \\
\hline - & $2 / 71$ & $\mathrm{P}, \mathrm{S}$ & Bucci et coll., 1975 \\
\hline Accadia & $1 / 5$ & S & - $\quad-$ \\
\hline Bovino & $1 / 10$ & $\mathrm{~s}$ & - \\
\hline Cagnano Varano & $1 / 18$ & $\mathrm{~S}$ & - \\
\hline Cerignola & $1 / 11$ & $\mathrm{P}, \mathrm{S}$ & - \\
\hline Faeto & $1 / 9$ & $\mathrm{P}, \mathrm{S}$ & - \\
\hline Isole Tremiti & $1 / 18$ & $\mathrm{~S}$ & - \\
\hline Manfredonia & $3 / 9$ & $\mathrm{P}, \mathrm{S}$ & - \\
\hline Orsara di Puglia & $1 / 11$ & $\mathrm{P}, \mathrm{S}$ & - \\
\hline Peschici & $1 / 8$ & $\mathrm{~S}$ & - \\
\hline Roseto Valfortore & $1 / 3$ & $\mathrm{P}, \mathrm{S}$ & - \\
\hline S. Giovanni Rotondo & $4 / 18$ & $\mathrm{~S}$ & - \\
\hline S. Paolo Civitate & $1 / 6$ & $\mathrm{P}, \mathrm{s}$ & - \\
\hline S. Severo & $2 / 10$ & $\mathrm{~S}$ & - \\
\hline Scannicandro Garganico & $2 / 12$ & $\mathrm{~S}(1 \mathrm{P})$ & $\ldots$ \\
\hline Torremaggiore & $4 / 27$ & $\mathrm{~S}(1 \mathrm{P})$ & - \\
\hline Trinitapoli & $1 / 18$ & $\mathrm{P}, \mathrm{S}$ & - \\
\hline Vieste & $2 / 9$ & $\mathrm{~S}$ & - $\quad-$ \\
\hline Ostuni & 1 & $\mathrm{P}, \mathrm{S} !$ & Données des auteurs \\
\hline S. Cesarea & 1 & $\mathrm{P}, \mathrm{S}$ ! & - _ \\
\hline Matino & 1 & $\mathrm{P}, \mathrm{S} !$ & Maroli et coll., 1983 \\
\hline \multicolumn{4}{|l|}{ Basilicate } \\
\hline Matera & $2 / 97$ & $\mathrm{P}$ & Tasselli et Colella 1960 \\
\hline - & $7 / 103$ & $\mathrm{P}$ & Colella et Casamassima, 1975 \\
\hline - & $4 / 60$ & $\mathrm{~S}(3 \mathrm{P})$ & Colella et coll. 1979 \\
\hline Aliano-Alianello & $7 / 54$ & $\mathrm{~S}(1 \mathrm{P})$ & - — \\
\hline Bernalda & $1 / 12$ & $\mathrm{~S} 1+1$ & - \\
\hline Grassano & $2 / 13$ & $S(1 P)$ & - - \\
\hline Grottole & $3 / 12$ & $\mathrm{~S}(2 \mathrm{P})$ & -- \\
\hline Miglionico & $2 / 7$ & $\mathrm{P}, \mathrm{S}$ & - - \\
\hline Montescaglioso & $6 / 15$ & $\mathrm{~S}(5 \mathrm{P})$ & - - \\
\hline Pisticci & $2 / 25$ & $\mathrm{P}, \mathrm{S}$ & - \\
\hline Policoro & $2 / 16$ & $\mathrm{~S}^{\prime}(1 \mathrm{P})$ & - - \\
\hline Pomarico & 1 & $\mathrm{P}, \mathrm{S}$ & $-\ldots$ \\
\hline Scanzano & $3 / 30$ & $\mathrm{~S}^{\prime}(1 \mathrm{P})$ & - \\
\hline Stigliano & $1 / 2$ & $\mathrm{P}, \mathrm{S}$ & - - \\
\hline Tricarico & $1 / \overline{9}$ & $\mathrm{P}, \mathrm{S}$ & -- \\
\hline Tursi & $4 / 51$ & $\mathrm{~S}$ & - - \\
\hline
\end{tabular}


Tableau I (suite)

\begin{tabular}{|c|c|c|c|}
\hline $\begin{array}{l}\text { Région } \\
\quad \text { Localité }\end{array}$ & $\begin{array}{l}\text { Nombre de } \\
\text { chiens } \\
\text { positifs* }\end{array}$ & $\begin{array}{c}\text { Type } \\
\text { de } \\
\text { test** }\end{array}$ & Bibliographie \\
\hline \multicolumn{4}{|l|}{ Calabre } \\
\hline Reggio Calabre (province) & 5 & $\mathbf{P}$ & Catarsini, 1972 \\
\hline - & 25 & $\mathrm{P}$ & Catarsini, 1977 \\
\hline Campo Calabre & 1 & $\mathrm{P}$ & Giugni, 1915 \\
\hline Catanzaro & 2 & $\mathrm{P}$ & Mazza, 1950 \\
\hline \multicolumn{4}{|l|}{ Sicile } \\
\hline Palerme & 1 & $\mathrm{P}$ & Jemma, 1912 \\
\hline - & $1 / 2$ & $\mathrm{P}$ & Caronia et Di Giorgio, 1914 \\
\hline - & $34 / 434$ & S & Mercurio, 1955 \\
\hline - & 3 & $\mathrm{P}$ & Catarsini, 1977 \\
\hline - & l & $\mathrm{P}, \mathrm{S} !$ & Données des auteurs \\
\hline - & $14 / 198$ & $\mathrm{~S}$ & Mansueto et coll., 1982 \\
\hline Carini & $\begin{array}{l}1+/ 190 \\
1\end{array}$ & $\stackrel{\mathrm{P}}{\mathrm{P}}$ & Jemma, 1912 \\
\hline Ustica & $23 / 62$ & $\mathrm{~S}$ & Mansueto et coll., 1982 \\
\hline Catane & $3 / 300$ & $\mathrm{P}$ & Pulvirenti, 1911 \\
\hline — & $4 / 181$ & $\mathrm{P}$ & Pantò, 1912 \\
\hline - & $6 / 100$ & $\mathrm{P}$ & Previtera, 1934 \\
\hline - & $6 / 82$ & $\mathbf{P}$ & Previtera et Bongiardo, 1948 \\
\hline - & $1 / 110$ & $\mathrm{P}$ & Reitano et coll., 1965 \\
\hline - & 21 & $\mathrm{P}$ & Catarsini, 1972 \\
\hline - & 70 & $\mathrm{P}$ & Catarsini, 1977 \\
\hline Messine & "quelques " & $\mathrm{P}$ & Bozzelli, 1936 \\
\hline - (ville) & 115 & $\mathrm{P}$ & Catarsini, 1972 \\
\hline - commune) & 59 & $\mathbf{P}$ & - \\
\hline - (province) & 14 & $\mathrm{P}$ & - $\quad-$ \\
\hline - (ville) & 60 & $\mathrm{P}$ & Catarsini, 1977 \\
\hline - (commune) & 55 & $\mathrm{P}$ & $-\quad-$ \\
\hline - (province) & 34 & $\mathrm{P}$ & - $\quad$ - \\
\hline Bordonaro & $27 / 33$ & $\mathrm{P}$ & Basile, 1910 \\
\hline - & $19 * * * *$ & $\mathrm{P}$ & Basile, 1913 \\
\hline Raguse & 2 & $\mathrm{P}$ & Catarsini, 1977 \\
\hline Syracuse & $\overline{7}$ & $\mathrm{P}$ & Catarsini, 1972 \\
\hline - & 13 & $\mathrm{P}$ & Catarsini, 1977 \\
\hline Sciacca & $9 / 77$ & $\mathrm{~S}$ & Mansueto et coll., 1982 \\
\hline Trapani & 3 & $P$ & Catarsini, 1977 \\
\hline
\end{tabular}

Sardaigne
Cagliari
Nuraminis
Pirri
Quartu
Selargiu
Sinnai
Oristano
Ales
Curcuris
Furtei
Genuri
Pompu
Zeppara di Ales

$\begin{array}{ll}3 & \mathrm{~S} \\ 1 & \mathrm{~S} \\ 1 & \mathrm{P}, \mathrm{S} ! \\ 2 & \mathrm{P}, \mathrm{S} ! \\ 1 & \mathrm{P}, \mathrm{S} ! \\ 2 & \mathrm{~S}(1 \mathrm{P}) ! \\ 1 & \mathrm{P} \\ 2 & \mathrm{~S}(1 \mathrm{P}) ! \\ 4 & \mathrm{~S}(2 \mathrm{P}) ! \\ 1 & \mathrm{P}, \mathrm{S} ! \\ 1 & \mathrm{~S}, \mathrm{~S} ! \\ 1 & \mathrm{P}, \mathrm{S} ! \\ 1 & \mathrm{P}, \mathrm{S} ! \\ 2 & \mathrm{~S}\end{array}$

Bettini et coll., 1983

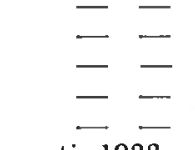

Cerruti, 1933

Bettini et coll., 1983

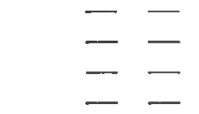

Bettini et coll., 1983 
TABLEAU I (suite)

\begin{tabular}{lcll}
\hline $\begin{array}{c}\text { Région } \\
\text { Localité }\end{array}$ & $\begin{array}{c}\text { Nombre de } \\
\text { chiens } \\
\text { positifs* }\end{array}$ & $\begin{array}{c}\text { Type } \\
\text { de } \\
\text { test** }\end{array}$ & \multicolumn{1}{c}{ Bibliographie } \\
\hline Sassari & "quelques " & $\mathrm{P}$ & Sotgia, 1921 \\
- & 1 & $\mathrm{P}$ & Pegreff et Doria, 1932 \\
- & 9 & $\mathrm{P}$ & Pegreff et Doria, 1934 \\
- & 16 & $\mathrm{P}$ & Carta, 1938 \\
- & 1 & $\mathrm{P}$ & Carta, 1939 \\
Alghero & 1 & $\mathrm{P}$ & Carta, 1942 \\
Olmedo & $2 / 41$ & $\mathrm{P}, \mathrm{S}$ & Arta, 1961 \\
S. Teresa di Gallura & 2 & $\mathrm{P}$ & Nieddu et Petruzzi, 1971 1980 \\
\hline
\end{tabular}

* Nombre de chiens positifs/Nombre de chiens examinés après des enquêtes épidémiologiques ou seulement Nombre de chiens positifs après des signalations de cas particuliers.

** $\quad \mathrm{P}=$ parasitologique $; \mathrm{S}=$ siérologique.

*** Tire faible à la fixation du complément $(1: 20)$, voir dans le texte.

**** 4 chiens positifs en 1910, 5 en 1911, 7 en 1912 et 3 en 1913 (Basile, 1913)

! Des souches de Leishmanies ont été obtenus.

- des examens parasitologiques et/ou sérologiques effectués dans des Instituts zooprophylactiques (pour lesquels l'anamnèse manque souvent) ;

- des examens parasitilogiques effectués auprès de Cliniques Universitaires ;

-- des enquêtes sur le terrain qui reflètent la véritable diffusion de la maladie (Mansueto et coll., 1982) dans 3 foyers de la Sicile.

Les informations que nous avons recueillies nous-mêmes concernent :

- des cas indiqués par des vétérinaires et dont on a pu connaître l'anamnèse (ces vétérinaires examinaient souvent des Chiens qui, résidant à Rome avaient passé l'été dans des régions d'endémie leishmanienne) ;

- des enquêtes dans des foyers bien définis où la presque totalité de la population canine a été examinée (Gradoni et coll., 1980 ; Pozio et coll., 1981a).

En ce qui concerne les cas de leishmaniose canine en Émilie, Pampiglione et coll. (1974) estiment que les renseignements apportés par Di Domizio (1955) sur Bologne et par Serra (1941) sur Modène, ne se réfèrent pas à des cas autochtones. Par ailleurs, les positivités sérologiques (1:20) qui ent été constatées dans la province de Bologne par Pampiglione et coll. (1974), en l'absence de signes cliniques, étaient trop basses pour certifier la présence de la maladie chez les Chiens.

En ce qui concerne le diagnostic, les techniques utilisées sont différentes selon le temps et les auteurs. De 1910 à 1948, on a utilisé exclusivement l'examen parasitologique, réalisé après nécropsie, principalement sur frottis de rate, foie et moelle osseuse. A partir de 1948, certains auteurs (Amalfitano et coll., 1948 ; Mercurio, 1955) 
ont utilisé des tests sérologiques, comme la formol-gélification et la lacto-gélification (tous deux ne sont pas considérés valables actuellement). La fixation du complément (FC) a été utilisée par Pampiglione et coll. (1974), par Gradoni et coll. (1980) comme test sérologique sur des Chiens d'Orbetello Scalo et Baccinello (Grosseto), et sur des sujets examinés à l'Institut Zooprophylactique du Latium et de la Toscane. En 1975, Bucci et coll. ont utilisé, outre la FC et l'immunodiffusion (ID), l'immunofluorescence indirecte (IF), mise en pratique ensuite par Colella et coll. (1979), Palarchi et coll. (1979), Lillini et coll. (1979), Polidori et coll. (1980) et par nousmêmes. En 1982, Mansueto et coll. ont utilisé le test micro-ELISA et la contreimmunoélectrophorèse (CIEP). La plupart des auteurs, en dehors de ceux cités ci-dessus, a continué à utiliser l'examen parasitologique, soit sur pièce nécropsique, soit sur biopsie de ganglion lymphatique ou d'ulcère et sur ponction sternale (Catarsini, 1977).

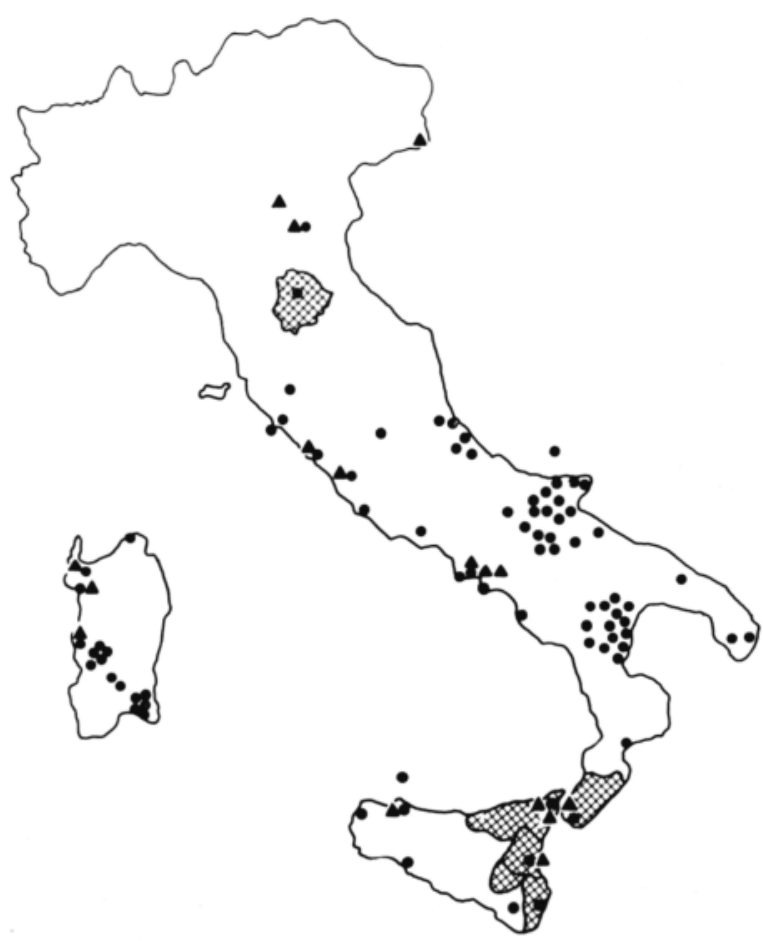

A Foyers localisés de leishmaniose canine de r91o à $194^{2}$

- Foyers localisés de leishmaniose canine de r 945 à 1983

$\$$ Aire où chaque cas signalé n'a pas été localisé

FIG. I. - Distribution de la leishmaniose canine de I9 Io à I983 en Italie 
Dans la figure 1, nous avons représenté la distribution des foyers de leishmaniose canine recensés avant-guerre et celle des foyers signalés depuis l'après-guerre jusqu'à maintenant. La nette augmentation, de 16 à 81 , doit être notée. Il est difficile d'établir si cette augmentation a un rapport avec une plus grande diffusion de la maladie. Catarsini (1977) signale une augmentation constante de la fréquence de la leishmaniose canine parmi les Chiens examinés à la Clinique Vétérinaire de Messine, avec une incidence annuelle croissante de $14 \%$ en 1972 à $25,5 \%$ en 1977. Toutefois, nous ne savons pas s'il s'agit d'un phénomène local ou d'un problème répandu également dans d'autres régions. En effet, il faut considérer que, de plus en plus, on s'adresse au vétérinaire pour soigner son Chien, et que la maladie est mieux connue des vétérinaires, grâce en particulier à l'utilisation des méthodes sérologiques. D'autre part, il faut prendre aussi en considération d'une part la facilité avec laquelle on déplace les Chiens d'une localité à l'autre, surtout en été, d'autre part l'augmentation des Chiens errants (Boitani et Fabbri, 1983). Ces deux facteurs peuvent avoir facilité, à cause de l'ubiquité des Phlébotomes vecteurs, l'installation de nouveaux foyers de la maladie dans le centre et le sud de l'Italie, du niveau de la mer à $600 \mathrm{~m}$ d'altitude (Biocca et coll., 1977).

Beaucoup d'auteurs ont isolé des Leishmanies chez les Chiens, par des méthodes in vivo (p. ex. Galassi, 1953) ou in vitro (p. ex. Adler et Theodor, 1935a). Les souches isolées n'ont cependant pas été maintenues longtemps. Au cours de nos travaux, nous avons obtenu des souches de Leishmanies au moyen d'inoculation in vivo (Hamsters) ou in vitro (Milleu de Tobie modifié par Evans (1978) du matériel prélevé de ganglions lymphatiques (Gramiccia et coll., 1979, 1980)). Ces souches sont conservées dans la cryobanque du Laboratoire de Parasitologie de l'Institut Supérieur de la Santé à Rome (Gramiccia et coll., 1983). Toutes les souches ont été caractérisées isoenzymatiquement comme étant Leishmania infantum, mais les 2 souches obtenues à Orbetellc Scalo et Baccinello (Grosseto) ont présenté une différence pour 3 enzymes par rapport au L. infantum de référence (Gramiccia et coll., 1982). Deux souches de Leishmanies ont été obtenues de Palarchi et coll. (1979) : elles proviennent de deux chiens de la province de Florence. Enfin, Lillini et coll. (1979) ont obtenu une souche isolée chez un Chien de Rome appartenant au groupe L. donovani (Schnur et coll., 1981).

\section{Conclusions}

Les récentes recherches de Bettini et coll. (1980), Pozio et coll. (1981b) et Gradoni et coll. (1983) ont impliqué le Rat noir (Rattus rattus) comme réservoir naturel de la leishmaniose viscérale dans la province de Grosseto. A Grosseto, Gradoni et coll. (1978) et Bettini et coll. (1980) ont découvert la maladie chez le Renard (Vulpes vulpes). Ceci rend encore plus complexe le cadre épidémiologique de la maladie et pourrait expliquer, peut-être, l'apparition chez les Chiens de cas sporadiques qui se produisent dans certaines localités. D'après les connaissances actuelles, le Chien est le principal réservoir de la parasitose. Toutefois, l'amélioration des condi- 
tions hygiéniques et sociales a amené une diminution des cas de leishmaniose viscérale humaine dans le Bassin méditerranéen. Par ailleurs, dans la même région, la fréquence des cas de leishmaniose canine est restée constante, et parfois même a augmenté (Lanotte et coll., 1974, 1978, 1979 ; Catarsini, 1977 ; Quilici et coll., 1979).

Remerciements. Nos plus sincères remerciements vont aux vétérinaires, les Drs M. Candutti (Rome), F. Contesso (Rcme), E. Lametti (Pescara), P. Madrucci (Grosseto) et M. Palumbo (Rome) pour leurs informations sur les cas suspects de leishmaniose canine.

\section{BIBLIOGRAPHIE}

Adler S., Theodor O. : Investigations on Mediterranean Kala-Azar VI Canine visceral leishmaniasis. Proc. Roy. Soc. London, I935a, I70, 402-4I2.

AdLeR S., Theodor O. : Investigations on Mediterranean Kala-Azar IX Feeding experiments. with Phlebotomus perniciosus and other species on animals infected with Leishmania infantum. Proc. Roy. Soc. London, 1935b, I16, 516-542.

Albano V. : Prime osservazioni di leishmaniosi canina nei comuni vesuviani della provincia di Napoli. Folia Med., I94 I, 27, 542-546.

amalfitano G., Benetazzo B., Cifarelli F., Gambini G., Servino V., Tarizzo M. : La leishmaniosi viscerale nella piana di Fondi (Latina) (Relazione delle prime ricerche epidemiologiche e cliniche). Arch. Ital. Sci. Med. Colon. Parassitol., I948, 19, I-I4.

Arru E., Pasqualini C. : Babesiosi e leishmaniosi del cane nella città di Sassari. Atti Soc. Ital. Sci. Vet., I97 I, $25,454-45^{8}$.

Basile C. : Alcune osservazioni sulla presenza di leishmanie dei cani. Atti R, Accad. Lincei Rend., I910, $5^{\circ}$ S., $19,15^{8-160 .}$

BASILE C. : I recenti studi sull'identità della leishmaniosi umana e canina del Mediterraneo. Policlinico Sez. Prat., 1913, 20, 1029-1032.

Bettini S., Pozio E., Gradoni L. : Leishmaniasis in Tuscany (Italy) II Leishmania from wild Rodentia and Carnivora in a human and canine leishmaniasis focus. Trans. R. Soc. Trop. Med. Hyg., I $980,74,77-83$.

Bettini S., Coni V., Gradoni L., Gramiccia M., Pozio E., Atzeni M. C. : Isolamento e tipizzazione enzimatica di Leishmania da cani in Sardegna. Atti XII Congr. Naz. Soc. Ital, Parassitologia, Como 28-30 giugno I983, Parassitologia (in press).

Biocca E.., Coluzzi A., Costantini R. : Distribution des différentes espèces de phlébotomes en Italie et transmission des leishmanioses et de quelques arboviroses. Écologie des leishmanioses. Colloques internationaux du C.N.R.S., 1977, n. 239.

Bortani L., FABBRI M. L. : Censimento dei cani in Italia con particolare riguardo al fenomeno del randagismo. Seminario : Il fenomeno del randagismo e la problematica ambientale, Cosenza 29 gennaio 1983.

Bozzelli R. : Su alcuni casi di leishmaniosi viscerale del cane osservati in Messina. Riv. Sanit. Sicil., 1936, 24, 896-874.

Bucci A., Puccini V., Casaglia O., Colella G. : Epizoologia della leishmaniosi in provincia di Foggia : la leishmaniosi del cane. Parassitologia, $1975,17,25-37$.

Caronia G., Di Grorgio G. : Sulla leishmaniosi spontanea nei cani di Palermo. Pathologica, I9I 4, 6. 208-209.

CARTA A. : Osservazioni morfologiche su milze di cani con leishmaniosi e con leishmaniosi e altre infestazioni associate. Nuovo Ercolani, I938, 16, 435-445.

Carta A. : Osservazioni morfologiche su midollo osseo, linfoghiandole e fegato di cani con leishmaniosi ed altre infezioni associate. Profilassi, I939, I2, 210-216.

Carta A. : Un caso di poliartrite cronica da Leishmania infantum osservato nel cane. Siudi Sassaresi, 1942, 20, 20I-2IO.

Carta A. : Primo focolaio di leishmaniosi canina identificato nella città di Sassari dopo la lotta antianofelica. Riv. Parassitologia, I961, 22, 89-92.

Catarsini O. : La leishmaniosi nel cane a Messina dal i959 al i971. Veterinaria, 1972, 21, 2 I I-2I9.

Catarsini $O$. : Incidenza della leishmaniosi del cane nell'attività ambulatoriale della Clinica Medica Veterinaria di Messina. Atti Soc. Ital. Sci. Vet., I977, 31, 486-488.

Cerruti G. : Su di un caso di leishmaniosi viscerale canina. Rapporti fra leishmaniosi umana e canina. Riforma Med., 1933, 49, 575.

Colella G., Casamassima E. : La leishmaniosi del cane in provincia di Matera. Vet. Ital., I975. 26. 393-399. 
Colella G., Bucci A., Puccini V., Casaglia O., la Salandra M. : Epizoologia della leishmaniosi in provincia di Matera : indagine sulla diffusione della malattia nel cane e sulla presenza di flebotomi. Clin. Vet., I979, 102, 35-4I.

Corradetri A., Sacca G., Neri I. : Studi epidemiologici sul Kala Azar nel promontorio garganico (Puglie, provincia di Foggia). Riv. Parassitologia, I 956, I7, 5-I0.

Di Domızı G. : Casi mortali di leishmaniosi viscerale canina autoctona in Bologna. Contributo allo studio anatomo-patologico e parassitologico. Zooprofilassi, 1955, 10, 399-414.

Evans D. A. : Kinetoplastida. In : Methods of cultivating parasites in vitro (Taylor A.E.R., Baker J. R. Eds) Academic Press Inc., London, N. Y., Pp. 55-88.

Franchini G. : Ricerche sui protozoi e sulle microfilarie nei cani in provincia di Bologna e di Ferrara. Pathologica, 1925, I7, I9-2I.

Galassi D. : Patogenicità per il Mesocricetus auratus e resistenza alle basse temperature di un ceppo di Leishmania isolato dal cane. Atti Soc. Ital. Sci. Vet., 1953, 7, 952-954.

GiUgNi F. : Alcuni tentativi di trasmissione della leishmaniosi canina. Ándamento clinico e dati necroscopici di un caso di leishmaniosi nel cane. Malar, Mal. Paesi Caldi, 191 5, 6, 77-8I.

Gradoni L., Pozio E., Bettini S., Gramiccia M. : Ricerche di leishmanie in carnivori selvatici della provincia di Grosseto. Ig. San. Publ., I978, 34, 257-268.

Gradoni L., Pozio E., Bettini S., Gramiccia M. : Leishmaniasis in Tuscany (Italy). (III) The prevalence of canine leishmaniasis in two foci of Grosseto Province. Trans. R. Soc. Trop. Med. Hyg., I $980,74,421-422$.

Gradoni L., Pozio E., Gramiccia M., Maroli M., Bettini S. : Leishmaniasis in Tuscany (Italy) : VII Studies on the role of the black rat, Rattus rattus, in the epidemiology of visceral leishmaniasis. Trans. R. Soc. Trop. Med. Hyg., I 983, 77, 427-43I.

Gramiccia M., Pozio E., Gradoni L., Bettini S. : Osservazioni sulla crescita in vivo e in vitro di vari isolati di Leishmania. Ig. San. Pubbl., I979, 35, I29-135.

Gramiccia M., Gradoni L., Pozio E., Betrini S.: Isolati di Leishmania. Ulteriori osservazioni. Ig. San. Pubbl, 1980, 36, 310-315.

Gramiccia M., Maazoun R., Lanotte G., Rioux J.-A., Le Blance S., Evans D. A., Peters W., Bettini S., Gradoni L., Pozio E. : Typage enzymatique de onze souches de Leishmania isolées en Italie continentale à partir de formes viscérales murines, canines et vulpines. Mise en évidence d'un variant enzymatique chez le Renard (Vulpes vulpes) et le chien. Ann. Parasitol. Hum. Comp., 1982, $57,527-53$ I.

Gramiccia M., Gradoni L., Pozio E. : Criobanca del Laboratorio di Parassitologia, Reparto di Protozoologia, Istituto Superiore di Sanità. Rapporto ISTISAN, $1983 / 8$.

Istituto Zooprofilattico Sperimentale del Lazio e della Toscana : Relazione dell'attività svolta nel 1980 .

Istituto Zooprofilattico Sperimentale del Lazio e della Toscana : Relazione dell'attività svolta nel I98I.

Jemma R. : Sulla leishmaniosi del cane nei dintorni di Palermo. Pathologica, I9 12, 4, 466-467.

LanotTe G., Rioux J. A., Croset H., Vollhard Y. : Dépistage de la leishmaniose canine. Stratégie d'enquête utilisée dans le foyer des Cévennes méridionales. In : Écologie des leishmanioses. Colloques Internationaux $d u$ C.N.R.S., $\mathrm{n}^{\circ}$ 239, Montpellier, I8-24 août I974. I I 7-I 28 .

Lanotte G., Rioux J. A., Croset H., Vollhardt Y. : Écologie des leishmanioses dans le Sud de la France. 9. Les méthodes d'échantillonnage dans le dépistage et l'analyse de l'enzootie canine. Ann. Parasitol. Hum. Comp., 1978, 53, 33-45.

Lanotte G., Rioux J. A., Croset H., Vollhardt Y.: Écologie des Leishmanioses dans le sud de la France. Io. Les formes évolutives de la leishmaniose viscérale canine. Élaboration d'une typologie bioclinique à finalité épidémiologique. Ann. Parasitol. Hum. Comp., 1979, 14. $277-295$.

Lillini E., Cavallina R., Piragino S., Aleandri M. : Indagini sulla leishmaniosi del cane nel territorio di Roma. Atti Soc. Ital. Sci. Vet., 1979, 33, $28 \mathrm{I}$.

Lucrezi G. : Reperti di leishmanie nei cani randagi di Napoli. Riv. Med. Trop. Studi Med. Indigena I940, 4, I9-23.

Mansueto S., Di Leo R., Miceli M. D., Quartararo P. : Canine leishmaniasis in three foci in Western Sicily. Trans. R. Soc. Trop. Med. Hyg., I982, 76, 565-566.

Marchesi F., Crainz F., Scapaticci R. : La leishmaniosi dei cani in Roma (Ricerche compiute nella stagione invernale e primaverile). Boll. Atti Accad. Med. Roma, I935, 60, 296-303.

Marchesi F., Crainz F., Scapaticci R. : Ricerche sulle variazioni stagionali della leishmaniosi dei cani in Roma. Arch. Ital. Sci. Med. Colon. Parassitol., 1935, I6, 805-8 2.

Mastropietro A. : Segnalazione del primo caso di leishmaniosi del cane a Campobasso. Atti Soc. Ital. Sci. Vet., 1953, 7, 894-898.

Mazza G. : Descrizione di un caso di leishmaniosi canina. Considerazioni sulla necessità di creare un piano organico di profilassi. Ig. San. Pubbl., I950, 6, 419-428.

Mercurio G. : Ricerche sulla leishmaniosi canina nell'agro palermitano. Atti Soc. Ital. Sci. Vet., I955, 9, 695-697.

Nardi E., De Pinto F., PAPA A. : La leishmaniosi del cane a Foggia. Zooprofilassi, i952, 7 , 46I-466. 
Nieddu A. M., Petruzzi V. : Leishmaniosi del cane in Sardegna : indagine epizoologica e considerazioni su due casi osservati ad Alghero. Atti $X$ Cong. Naz. Soc. It. Parassitologia, Alghero 20-33 maggio, Parassitologia, 1980, 22, 335.

Palarchi M., Lillini E., Cavallini R., Zanchi R., Tasselli E. : Indagini sulla leishmaniosi del cane nel territorio di Firenze. Atti Soc. Ital. Sci. Vet., 1979, 33, 280.

Pampiglione S., La Placa M., Schlick G. : Studies on mediterranean leishmaniasis I. An outbreak of visceral leishmaniasis in northern Italy. Trans. R. Soc. Trop. Med. Hyg., I974, 68, 349-359.

Pampigrione S., Bettini S. : Bibliografia Italiana della leishmaniosi dalle origini al i98o. Ann. Ist. Super. Sanità, I98I, I7, 2-I50.

Panto V. : La leishmaniosi spontanea del cane a Catania. Gazz. Int. Med. Chir. Ig. Inter. Profess., I 9 I $2, I 5,324-325$.

Pegreffi G., Doria C. : Su di un caso di leishmaniosi del cane. Studi Sassaresi, I932, II, $249-253$.

Pegreffi G., Doria C. : Osservazioni cliniche sulla leihsmaniosi canina in Sardegna. I Congr. Med. Regionale Sardo, Maggio I934, I-8.

Persechino $S$., Agresti A. : Su di un caso di leishmaniosi canina osservato nella città di Napoli. Acta Med. Vet., 1973, 19, 233-243.

Polidori A. G., Vitellozzi G., Agostinelli A., Principato M. : Indagine sulla fauna parassitaria in cani della città di Terni. Atti X Cong. Naz. Soc. It. Parassitol., Alghero 20-23 maggio, Parassitologia, 1980, 22, 349-350.

Pozio E., Gradoni L., Bettini S., Gramiccia M. : Leishmaniasis in Tuscany (Italy) : VI Canine leishmaniasis in the focus of Monte Argentario (Grosseto). Acta Trop., I981a, 38, 383-393.

Pozio E., Gradoni L., Bettini S., Gramiccia M. : Leishmaniasis in Tuscany (Italy) : V Further isolation of Leishmania from Rattus vatius in the province of Grosseto. Ann. Trop. Med. Pavasitol., I981 b, 75, 393-395.

Previtera A. : La leishmaniosi viscerale canina in Catania. Policlinico Sez. Pratica, I934, 4I, 65.

Previtera A., Bongiardo A. : Nuovi dati sulla leishmaniosi canina a Catania. Riv. Ital. Ig., I $948,8,3$ I $2-3$ I 8 .

Pulvirenti G. : La leishmaniosi del cane a Catania. Pathologica, I9I I, 3, 205-206.

Quilici M., Dunan S., Duman H., Franck J., Gambarelli F., Ranque J. : Persistance de la leishmaniose viscérale dans le sud-est de la France et fréquence marquée de l'affection à l'âge adulte a propos d'observations récentes. Bull. Soc. Pathol. Exot., I979, 72, I I 8-I 24.

Reitano G., Pavone L., Saporito N. : Indagini sulla diffusione della leishmaniosi canina a Catania. Riv. Pediatr. Sicil., I965, 20, 253-258.

SANGIorgi G. : Leishmaniosi spontanea dei cani a Torino. Pathologica, 19I I, 3, 699-70o.

Schnur F., Chance M. L., Ebert F., Thomas S. C., Peters W. : The biochemical and serological taxonomy of visceralizing Leishmania. Ann. Trop. Med. Parasit., I98I, 75, I3I-I 44.

Serra G. : Quoted by Sirotti, 1934, I94I.

Silvagni $\mathrm{T}$. : Terapia della leishmaniosi del canc. Vet. Ital., 1955, 6. 52-57.

Sirotti R. : La leishmaniosi nel Modenese. Cenni epidemiologici e clinici. Clinica Pediatrica, 1954, 36, $219-233$

Sotgia G. M. : Leishmaniosi canina in Sardegna. Ig. Mod., I921, I4, 308-31o.

TAMPONI M. : Contributo casistico alla questione dei rapporti fra leishmaniosi canina e leishmaniosi cutanea umana. G. Ital. Dermatol. Sifilol., I935, 74, 324-328.

Tasselli E., Colella G. : Leishmaniosi nel cane e nell'uomo in provincia di Matera. Parassitologia, $1960,2,321-325$.

Venturoli M. : Aspetti clinici della leishmaniosi nel cane. C. Mal. Infett. Parassit., 1982, 34, I 524-I 526 .

Vlachi G. : I a leishmaniosi canina in Trieste e provincia. Arch. Ital. Sci. Med. Colon., I936, I7,

Von SCHLOTKE B. : Leishmaniasis (Kala-Azar) bei Hunden in Bayern. Berl. Münch. Tierärztl. Wschr., 1975, 88, 70-73.

Zechini M. : Leishmaniosi cutanea del cane di origine autoctona in Italia. Ann. Patol. Trop. Parassitol. Modena, I942, 3, 29-36. 